\title{
Phase-Sensitive Sum-Frequency Generation Measurements Using a Femtosecond Nonlinear Interferometer
}

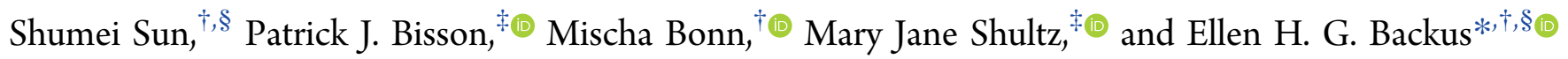 \\ ${ }^{\dagger}$ Max Planck Institute for Polymer Research, Ackermannweg 10, 55128 Mainz, Germany \\ ${ }^{\ddagger}$ Laboratory for Water and Surface Studies, Chemistry Department, Tufts University, Medford, Massachusetts 02155, United States \\ ${ }^{\S}$ Department of Physical Chemistry, University of Vienna, Währinger Str. 42, 1090 Wien, Austria
}

\section{Supporting Information}

ABSTRACT: Phase-sensitive sum-frequency spectroscopy is a unique tool to interrogate the vibrational structure of interfaces. A precise understanding of the interfacial structure often relies on accurately determining the phase of $\chi^{(2)}$, which has recently been demonstrated using a nonlinear interferometer in conjunction with a frequency-scanning picosecond laser system. Here, we implement nonlinear interferometry using a femtosecond laser system for broadband sumfrequency generation. The phase of the vibrational response from a self-assembled monolayer of octadecanethiol on gold is determined using the nonlinear femtosecond interferometer. The results are compared to those obtained using the more traditional heterodyne-detected phase measurements. Both methods give a similar phase spectrum and phase uncertainty. We also discuss the origin of the phase uncertainties and provide guidelines for further improvement.

\section{INTRODUCTION}

Sum-frequency vibrational spectroscopy (SFVS) has emerged as one of the main tools for characterizing molecular structure and dynamics at interfaces because of its high surface sensitivity and molecular specificity. ${ }^{1-7}$ However, in a conventional homodyne SFVS measurement, the SF signal is proportional to the square of the complex second-order nonlinear susceptibility. Analysis of the intensity spectrum through fitting can be ambiguous as the outcome of the fitting procedure is rarely unique. ${ }^{8,9}$ Thus, the phase information is often lost in a homodyne SF measurement. Therefore, the direct measurement of $\operatorname{Im} \chi^{(2)}$ or, equivalently, the phase of $\chi^{(2)}$ is frequently desirable. ${ }^{10,11}$

Since the seminal papers reporting the phase measurement methodology published by Shen et al., ${ }^{12,13}$ the technique of phase-sensitive sum-frequency vibrational spectroscopy (PSSFVS) has greatly developed in the past ten years, ${ }^{12-21}$ and phase accuracy has been a prime concern. Based on the existing beam configurations, one origin of phase uncertainty is the height difference between the sample surface and the reference surface. To improve the phase accuracy, Shen and co-workers adopted a collinear beam geometry. ${ }^{12,13}$ The collinear geometry greatly reduces the phase dependence on the height difference between the real sample and the reference sample because the path length is experienced by both sum frequency beams generated from the local oscillator (LO) and real sample or reference. ${ }^{22}$ Tahara and his co-workers adopted a noncolinear beam configuration but implemented a height

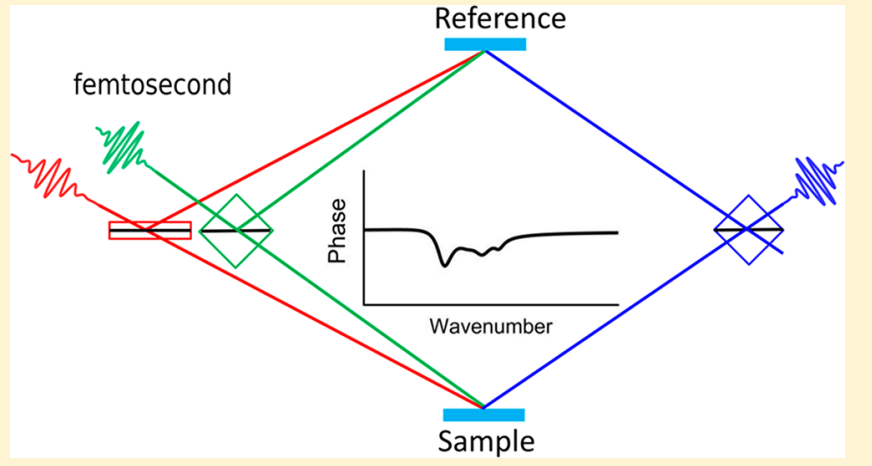

sensor in the setup, connected to a feedback system to monitor and correct the height of the sample in situ during a measurement. ${ }^{23}$ The height accuracy can be controlled within $\pm 1 \mu \mathrm{m}$. Martin Wolf and his co-workers developed a timedomain spectrometer, which combined collinear beam geometry and simultaneous referencing. This design also greatly reduces phase uncertainty. ${ }^{19}$

Although configurations differ depending on the laser system or the sample of interest, the underlying principle is the same: A phase measurement requires interference between two signals. In many setups, the phase measurement is termed a "heterodyne measurement", and a SF signal generated from what is referred to as the "local oscillator" (LO) interferes with the SF signal from a phase reference. Subsequently or simultaneously, the LO signal interferes with that of the sample of interest. Combining the two allows one to deduce the phase of the sample of interest.

It has proven challenging to elucidate details of the spectral shape of $\operatorname{Im} \chi^{(2)}$ in a completely unambiguous manner. This is related to the experimental challenges associated with determining the reference phase reliably and is well illustrated by the recent discussion of the presence-or not-of a positive peak in the SFG response of the $\mathrm{O}-\mathrm{H}$ stretch mode at the water-air interface at low frequencies. ${ }^{24,25}$ While this

Received: January 30, 2019

Revised: $\quad$ March 6, 2019

Published: March 6, 2019 
controversy for the water-air interface seems to have been resolved, the same controversy now exists for the ice surface, where different versions of the phase spectrum have been reported. ${ }^{26,27}$ Hence it is clear that a novel way of determining the phase of the SFG spectrum should be very helpful.

Recently, some of us have reported the development of a nonlinear interferometer scheme. ${ }^{17,28}$ The nonlinear interferometer operates somewhat differently. In normal operation, the setup involves monitoring the interference between two identical, phase reference materials, called reference and a "calibration sample". Since both reference and calibration samples have the same phase, the nonlinear interferometer operating point can be set from the interference. Subsequently, the calibration sample is removed and replaced by the sample of interest. The sample signal interferes with the referencethe phase standard-to directly deduce the sample phase.

So far, this nonlinear interferometer design has only been demonstrated using a picosecond laser system. ${ }^{17,28}$ In the SFG community, an increasing number of experimental setups are based on a femtosecond laser system because a femtosecond laser system offers a broad IR spectrum, high peak intensity, and high repetition rate, which allows reducing the acquisition time. Here, we implemented the interferometer with a femtosecond laser system and demonstrated it by comparing the obtained phase spectrum with the one obtained using the heterodyne phase-measurement configuration. ${ }^{14,23,29}$

\section{EXPERIMENT}

Experiments were performed on two different setups, both using a femtosecond Ti:Sapphire amplifier laser (SpectraPhysics) with an output of $5 \mathrm{~W}$ at $800 \mathrm{~nm}$ wavelength. The pulse duration of the laser is $50 \mathrm{fs}$ with a repetition rate of 1 $\mathrm{kHz}$. Roughly $2 \mathrm{~W}$ is used to pump an optical parametric oscillator to generate signal and idler beams (TOPAS). Subsequently, the signal and idler beams are sent to a difference frequency generator (crystal: $\mathrm{AgGaS}_{2}$ ), producing broad IR with a bandwidth around $400 \mathrm{~cm}^{-1}$ with a pulse energy around $5 \mu \mathrm{J}$. Another roughly $1 \mathrm{~W}$ of the laser output goes through an etalon to generate the narrowband (with just $15 \sim 25 \mathrm{~cm}^{-1}$ line width), temporally lengthened, $800 \mathrm{~nm}$ wavelength pulse of $\sim 20 \mu \mathrm{J}$ used as the visible beam in the SFVS process. A self-assembled monolayer of octadecanethiol (ODT) on gold was prepared by immersing a bare gold mirror (Thorlabs) in dilute (1 $\mathrm{mM})$ ODT solutions in absolute ethanol overnight. The sample was rinsed with absolute ethanol to remove the residual adsorbates and subsequently dried with nitrogen. ${ }^{30,31}$ The exact same sample was measured on both setups directly after each other, making a direct comparison between the two setups possible.

The layout of the nonlinear interferometer as shown in Figure 1a is similar to that described previously. ${ }^{17,28}$ Visible excitation, depicted with the green line, consists of $800 \mathrm{~nm}$; infrared excitation is depicted with the red line. White light (WL) plays an important role in repositioning the sample. ${ }^{17,28}$ The green laser beam is split at the BS. The split beams traverse paths $\mathrm{B}^{\prime}, \mathrm{B}, \mathrm{D}^{\prime}$, and $\mathrm{D}$, respectively, and combine at the $\mathrm{BC}$. The combination generates interference that is used in a feedback control system, so it is also referred to as the tracker laser. The alignment and operating procedures for the femtosecond laser system are similar to the picosecond laser system described in refs 15 and 18, with one difference. In the picosecond configuration, the tracker laser and SF alignment laser are a $\mathrm{He}-\mathrm{Ne}$ laser with wavelength of $633 \mathrm{~nm}$. For the
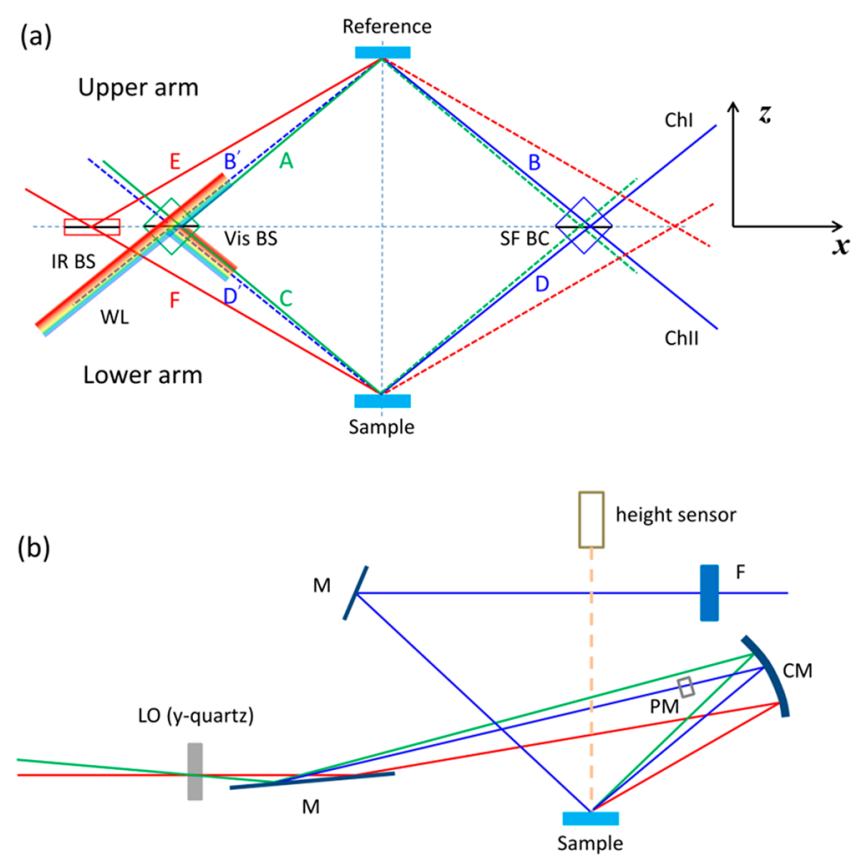

Figure 1. Layout of the nonlinear interferometer (a) and heterodyne phase measurement (b). Nonlinear interferometer: IR BS and Vis BS are the infrared and visible beam splitter, respectively; SF BC is the SF beam combiner; A, E, and B represent the optical paths of the Vis, IR, and SF beams for the upper arm, respectively; C, F, and D are the optical path of the Vis, IR, and SF beams for the lower arm. B' and $D^{\prime}$ are the mirror optical paths of $\mathrm{B}$ and $\mathrm{D}$, respectively. The colorful beam is white light (WL). A divergent green beam (not shown) follows the same paths as the WL: $\mathrm{B}^{\prime}, \mathrm{B}, \mathrm{D}^{\prime}$, and D. A collimated green alignment beam (not shown) also traverses $\mathrm{B}^{\prime}, \mathrm{B}, \mathrm{D}^{\prime}$, and $\mathrm{D}$ and also serves as a tracker beam. In panel (b), M, PM, CM, and F are mirrors, a phase modulator, concave mirror, and filter, respectively.

femtosecond setup, this is not suitable because the wavelength of $\mathrm{He}-\mathrm{Ne}$ falls into the SF bandwidth $(600-700 \mathrm{~nm})$. Consequently, in this implementation, all alignment and tracker lasers are diode lasers with a wavelength of $532 \mathrm{~nm}$.

As shown in Figure 1a, the SFs from the reference and the sample are combined at the BC. The SF signal can be detected in two channels. In the current measurement, only one channel (ChI) is collected, and the sum-frequency signal with $p p p$ (the $\mathrm{SF}$, Vis, and IR are $p$-polarized) polarization combination is used. The collected SF signal can be expressed as

$$
I=I_{\mathrm{s}}+I_{\text {ref }}+2 M \sqrt{I_{\mathrm{s}} I_{\text {ref }}} \cos \left(\delta_{\mathrm{s}}-\delta_{\text {ref }}+\delta_{\mathrm{I}}\right)
$$

where $I_{s}$ and $I_{\text {ref }}$ are the homodyne SF intensity from sample and reference detected separately in ChI. $\delta_{\mathrm{s}}$ and $\delta_{\text {ref }}$ denote the phases of the sample and reference, respectively (please note that for $\delta_{\mathrm{s}}$ and $\delta_{\text {ref }}$ in the equation the phase is not the pure phase from $\chi^{(2)}$ from the sample or reference; actually, in both cases, it also includes the phase from the Fresnel Factors); $\delta_{\mathrm{I}}$ is the instrument phase caused by the difference in the optical path between the lower and the upper paths; the quantity $M$ is the interference range; and $\delta_{\mathrm{I}}$ and $M$ can be determined when the same material as reference is placed at the sample position, which is termed a calibration sample. In our measurement, bare gold serves as the reference. Therefore, during alignment and calibration, another bare gold is placed at the sample position; it is the calibration sample. Because the reference and the calibration samples are the same, their phases are the same. Equation 1 becomes 


$$
I=I_{\text {cal-sample }}+I_{\text {ref }}+2 M \sqrt{I_{\text {cal-sample }} I_{\text {ref }}} \cos \left(\delta_{\mathrm{I}}\right)
$$

The instrument phase, $\delta_{\mathrm{I}}$, is selected by the operator; it is controlled by shifting the BC. At the maximum of the SF interference, $\delta_{\mathrm{I}}=0^{\circ}$, and at the midpoint, $\delta_{\mathrm{I}}= \pm 90^{\circ}$. The plus sign applies if the $\mathrm{BC}$ is shifted up and the minus sign if it is shifted down. This is termed setting the operating point. A near $-90^{\circ}$ instrument operating point aids in distinguishing positive and negative phases. $M$ is obtained by determining the maximum and minimum interference signal $I_{\max }$ and $I_{\min }$. As $\cos \left(\delta_{\mathrm{I}}\right)$ equals 1 and -1 in these cases, one obtains $M=\frac{I_{\max }-I_{\min }}{4 \sqrt{I_{\text {cal-sample }} I_{\text {ref }}}} . M(0 \leq M \leq 1)$ has several contributions including beam geometric overlap at the combiner. The optical paths $\mathrm{A}-\mathrm{D}$ are balanced using linear interferometry; the balance is maintained with the feedback control.

\section{RESULTS AND DISCUSSION}

After calibration, i.e., determining $M$ and $\delta_{\mathrm{I}}$, the sample (ODT/gold) replaces the gold calibration sample as described by Shultz et al. ${ }^{17,28}$ The green curve in Figure 2 shows the

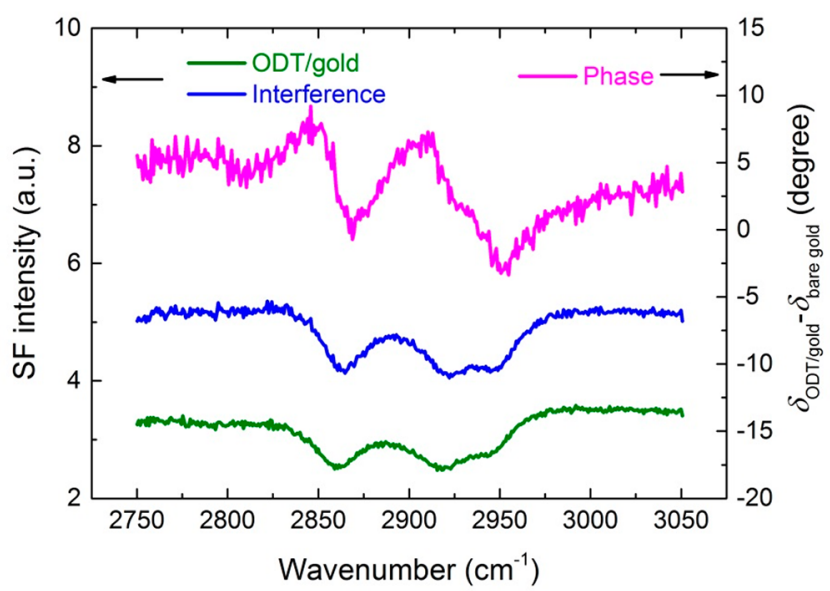

Figure 2. ODT/gold sample homodyne spectrum (green line) and the interference intensity (blue line) between ODT/gold and bare gold. The magenta line is the relative phase spectrum of ODT/gold vs bare gold.

intensity spectrum $I_{s}$ (i.e., homodyne spectrum) of the ODT/ gold sample obtained by blocking the signal from the reference. The blue curve depicts the interference signal $I$ (including the homodyne signals from sample and reference and their cross-term contribution) with the operating point of the interferometer close to $-90^{\circ}$. As $I_{\text {ref }}$ is also separately measured and $M$ and $\delta_{\mathrm{I}}$ are known from the calibration, we can extract $\delta_{\mathrm{ODT} / \text { gold }}-\delta_{\text {bare gold }}$ using eq 1 . The resulting phase spectrum is shown in magenta in Figure 2. The gold sample substrate is known to produce a nonresonant signal containing a nonresonant phase. The nonresonant phase of the ODT/ gold is expected to be different from that of bare gold as the phase is expected to shift with adsorption of a monolayer since the $\chi^{(2)}$ response of gold is largely due to the surface states. $^{31-34}$ The surface states are directly involved in binding the SAM to the surface. Assuming that the nonresonant response of the ODT/gold surface is constant across the $2750-3050 \mathrm{~cm}^{-1}$ band, the measured phase of ODT/gold can be estimated from the data in Figure 2. Relative to the phase of bare gold, the phase of the nonresonant part is about $5^{\circ}$ (average of the baseline on the left and right sides of the resonances), and the phase of the resonant part only varies within a few degrees in the measured frequency window. The small phase change upon passing through the vibrational resonances is a result of the large nonresonant amplitude. The relationship between the phase and the amplitude ratio of resonance/nonresonance is discussed in detail by Hore et al. ${ }^{35}$

We also performed the phase measurement with the heterodyne setup (the layout of the setup is depicted in Figure $1 \mathrm{~b}$ ). Details of the analysis procedure can be found in ref 12 . The result is plotted together with that obtained from the interferometer in Figure 3. The main phase features of

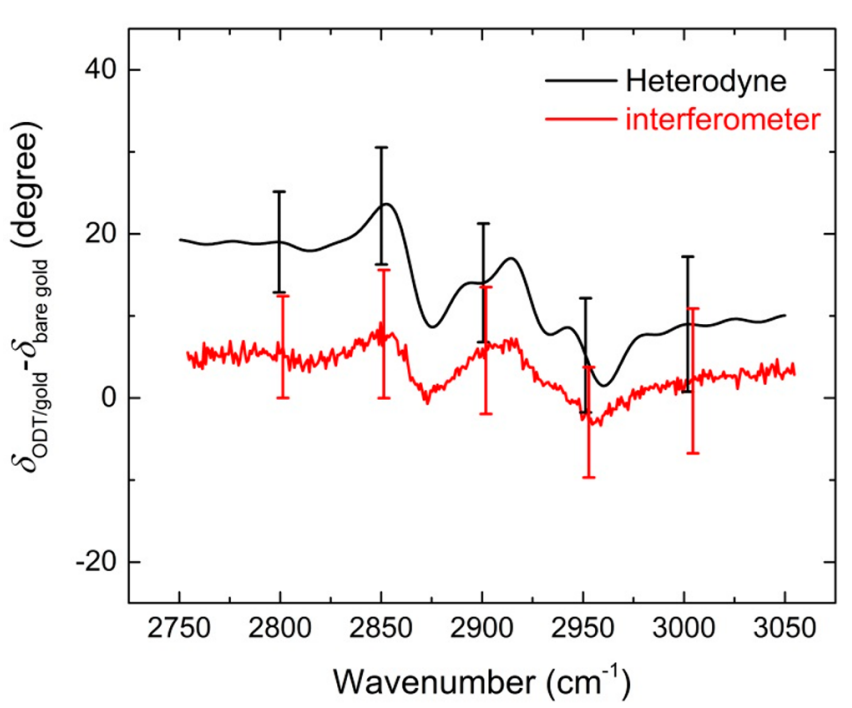

Figure 3. Comparison of the relative phase spectrum of ODT/gold vs gold obtained from the nonlinear interferometer measurement (red curve) and heterodyne measurement (black curve). The error bars, depicted only every $\sim 50 \mathrm{~cm}^{-1}$ for clarity, illustrate the standard deviation of several measurements in which we removed and replaced the sample in the measurement position.

ODT/gold obtained from the two different setups are similar. The dips and peaks between 2800 and $3000 \mathrm{~cm}^{-1}$ originate from the $\mathrm{C}-\mathrm{H}$ vibrations of the ODT layer. The higher $\mathrm{S} / \mathrm{N}$ of the spectrum obtained with the heterodyne measurement originates from the data processing. In the analysis, Fourier transformation with window filtering is used. The small vertical shift between the two data sets originates from measuring different spots on the slightly heterogeneous sample. The error bars on the curves in Figure 3 illustrate the variation between experiments in which we removed and replaced the sample in the sample position.

In the following, we will discuss the possible origins of the phase uncertainty for the two methods. In the heterodyne measurement, the height of the sample position and the reference is determined by a height sensor with a height uncertainty $d= \pm 1 \mu \mathrm{m}$. The incident angle of the Vis and the IR beams in our heterodyne setup is $47^{\circ}$ and $62^{\circ}$ with respect to the surface normal, respectively. Then the phase uncertainty i s give n b y $\left(\frac{2 \pi d}{\lambda_{\mathrm{Vis}}} \cos \theta_{\mathrm{Vis}}+\frac{2 \pi d}{\lambda_{\mathrm{IR}}} \cos \theta_{\mathrm{IR}}-\frac{2 \pi d}{\lambda_{\mathrm{SF}}} \cos \theta_{\mathrm{SF}}\right) 180 / \pi \sim \pm 5^{\circ}(\mathrm{s} \mathrm{e} \mathrm{e}$ the derivation details in SI), more or less in agreement with the experimental error bar in Figure 3. For the heterodyne measurement, the phase reproducibility can be improved by 
decreasing the angle between the Vis and IR beams. As shown in Figure 4, the phase uncertainty becomes vanishingly small for very small angles. ${ }^{22}$

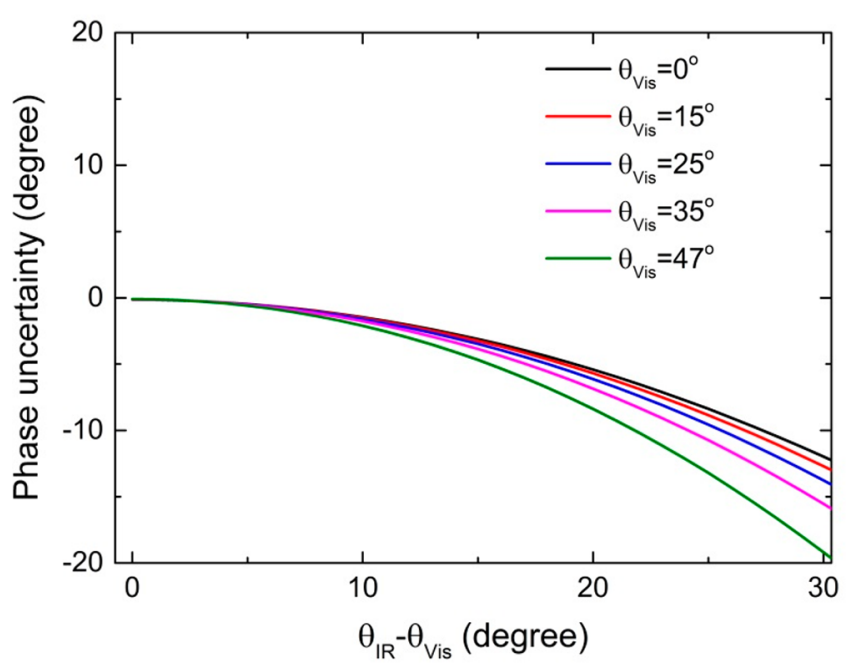

Figure 4. Phase uncertainty caused by the sample position changed by $1 \mu \mathrm{m}$ at different beam geometries in heterodyne phase measurement.

In the interferometer, the sample can be placed in a much more precise way than in the heterodyne measurement by taking advantage of the white light fringes and tracker beam interferogram. However, the phase is much more sensitive to a height difference because of the separated arms configuration. Referring to Figure 1a, in the procedure of sample placement, the recovery of the white light fringe and tracker beam interferogram allows us to get $\mathrm{B}^{\prime}+\mathrm{B}$ equals $\mathrm{D}^{\prime}+\mathrm{D}$. However, for the phase of the SFG signal, it is important that the phase difference between the lower and upper arm including the IR, Vis, and SFG path, $\left(k_{1} C+k_{2} F+k_{3} D\right)-\left(k_{1} A+k_{2} E+k_{3} B\right)$, is the same for the real sample and calibration sample. As the optical paths of $\mathrm{E}$ and $\mathrm{F}$ are not incorporated in the feedback control system, there is no compensation possible if the position of the IR BS is changed. Moreover, the position compensation in the linear interferometer through the feedback control can only partly balance the SFVS phase because of the fact that the frequencies and beam paths between Vis and SF are different. Table 1 shows the phase change caused by a vertical drift over a distance $d$ for different elements in the interferometer, keeping all other components at fixed height.

In our current lab environment, the temperature change is about $0.2{ }^{\circ} \mathrm{C}$ during sample replacement. This temperature change could induce a position change of the optics estimated to be $50 \mathrm{~nm}$. If the position of the IR BS changes by $50 \mathrm{~nm}$, the phase change amounts to $-\frac{4 \pi d}{\lambda_{\mathrm{IR}}} \cos \theta_{\mathrm{IR}} \frac{180}{\pi} \sim-5^{\circ}$ accordingly. If the position of the Vis $\mathrm{BS}$ changes $50 \mathrm{~nm}$, the $\mathrm{SF} B C$ compensates it by changing its position by $-50 \mathrm{~nm}$. A phase change of $\left(-\frac{4 \pi d}{\lambda_{\mathrm{Vis}}} \cos \theta_{\mathrm{Vis}}+\frac{4 \pi d}{\lambda_{\mathrm{SF}}} \cos \theta_{\mathrm{SF}}\right) 180 / \pi \sim 5^{\circ}$ will thus be introduced. Besides the position change of the optics induced by temperature variation, another possible origin of phase uncertainty might come from alignment errors (for example, in recovering the white light fringe by replacing the sample) yet to be identified.

Although the interferometer can recover the position of the sample with interferometric accuracy, it remains more sensitive to temperature changes than the heterodyne measurement. Hence, to get an interferometer with a stable phase, one needs to reduce the temperature fluctuation as much as possible during the measurement, especially in the sample replacement procedure. Combining automated sample replacement (preventing body heat from affecting the measurements) with good temperature control could overcome this problem. Although the alignment of the nonlinear interferometer is more sensitive to temperature fluctuations, there are several advantages. The separation of reference and sample arms supports a wide choice of the phase reference, and the splitting ratio can be chosen to balance the sample and reference SF intensities, enabling identification of weak signals.

\section{CONCLUSION}

A nonlinear interferometer designed for phase measurement has been demonstrated using femtosecond laser pulses by measuring the phase of the $\mathrm{CH}$ stretch vibrations of ODT/ gold. The consistent results from the nonlinear interferometer and heterodyne setups indicate that the nonlinear interferometer works well with a femtosecond laser system. Comparable phase accuracy has been demonstrated in our current lab environment.

\section{ASSOCIATED CONTENT}

\section{S Supporting Information}

The Supporting Information is available free of charge on the ACS Publications website at DOI: 10.1021/acs.jpcc.9b00861.

Derivation of the phase change due to height change for heterodyne measurement and nonlinear interferometer (PDF)

\section{AUTHOR INFORMATION}

\section{Corresponding Author}

*E-mail: backus@mpip-mainz.mpg.de.

ORCID

Patrick J. Bisson: 0000-0002-4985-3077

Mischa Bonn: 0000-0001-6851-8453

Mary Jane Shultz: 0000-0001-5625-1675

Ellen H. G. Backus: 0000-0002-6202-0280

\section{Notes}

The authors declare no competing financial interest.

Table 1. Phase Change Caused by a Vertical Drift over Distance $d$ for Several Elements (See the Derivation Details in SI) in the Interferometer Setup ${ }^{a}$

$\begin{array}{ccccc}\text { optical element } & \text { IR BS } & \text { Vis BS } & \text { SF BC } & \text { LO } \\ \text { phase change }(180 / \pi)^{\circ} & -\frac{4 \pi d}{\lambda_{\mathrm{IR}}} \cos \theta_{\mathrm{IR}} & -\frac{4 \pi d}{\lambda_{\mathrm{Vis}}} \cos \theta_{\mathrm{Vis}} & -\frac{4 \pi d}{\lambda_{\mathrm{SF}}} \cos \theta_{\mathrm{SF}} & \sum_{i} \frac{2 \pi d}{\lambda_{i}} \cos \theta_{i}\end{array}$

$a_{i}$ represents the IR, Vis, and SF beam. 


\section{ACKNOWLEDGMENTS}

This work was funded by an ERC Starting Grant (Grant No. 336679). MJS and PJB acknowledge the US National Science Foundation, grant number CHE1565772.

\section{REFERENCES}

(1) Shen, Y. Surface Properties Probed by Second-Harmonic and Sum-Frequency Generation. Nature 1989, 337, 519-525.

(2) Eisenthal, K. Liquid Interfaces Probed by Second-Harmonic and Sum-Frequency Spectroscopy. Chem. Rev. 1996, 96, 1343-1360.

(3) Richmond, G. Molecular Bonding and Interactions at Aqueous Surfaces as Probed by Vibrational Sum Frequency Spectroscopy. Chem. Rev. 2002, 102, 2693-2724.

(4) Shen, Y. R.; Ostroverkhov, V. Sum-Frequency Vibrational Spectroscopy on Water Interfaces: Polar Orientation of Water Molecules at Interfaces. Chem. Rev. 2006, 106, 1140-1154.

(5) Lu, X.; Zhang, C.; Ulrich, N.; Xiao, M.; Ma, Y.-H.; Chen, Z. Studying Polymer Surfaces and Interfaces with Sum Frequency Generation Vibrational Spectroscopy. Anal. Chem. 2017, 89, 466489.

(6) Nihonyanagi, S.; Yamaguchi, S.; Tahara, T. Ultrafast Dynamics at Water Interfaces Studied by Vibrational Sum Frequency Generation Spectroscopy. Chem. Rev. 2017, 117, 10665-10693.

(7) Cyran, J. D.; Backus, E. H.; Nagata, Y.; Bonn, M. Structure from Dynamics: Vibrational Dynamics of Interfacial Water as a Probe of Aqueous Heterogeneity. J. Phys. Chem. B 2018, 122, 3667-3679.

(8) Busson, B.; Tadjeddine, A. Non-Uniqueness of Parameters Extracted from Resonant Second-Order Nonlinear Optical Spectroscopies. J. Phys. Chem. C 2009, 113, 21895-21902.

(9) Yang, W.-C.; Hore, D. K. Broadband Models and Their Consequences on Line Shape Analysis in Vibrational Sum-Frequency Spectroscopy. J. Chem. Phys. 2018, 149, 174703.

(10) Shen, Y. Phase-Sensitive Sum-Frequency Spectroscopy. Annu. Rev. Phys. Chem. 2013, 64, 129-150.

(11) Tian, C.; Shen, Y. Recent Progress on Sum-Frequency Spectroscopy. Surf. Sci. Rep. 2014, 69, 105-131.

(12) Ji, N.; Ostroverkhov, V.; Tian, C. S.; Shen, Y. R. Characterization of Vibrational Resonances of Water-Vapor Interfaces by Phase-Sensitive Sum-Frequency Spectroscopy. Phys. Rev. Lett. 2008, 100, No. 096102.

(13) Ji, N.; Ostroverkhov, V.; Chen, C.-Y. Phase-Sensitive SumFrequency Vibrational Spectroscopy and Its Application to Studies of Interfacial Alkyl Chains. J. Am. Chem. Soc. 2007, 129, 10056-10057.

(14) Nihonyanagi, S.; Yamaguchi, S.; Tahara, T. Direct Evidence for Orientational Flip-Flop of Water Molecules at Charged Interfaces: A Heterodyne-Detected Vibrational Sum Frequency Generation Study. J. Chem. Phys. 2009, 130, 204704.

(15) Fu, L.; Chen, S.-L.; Wang, H.-F. Validation of Spectra and Phase in Sub-1 $\mathrm{Cm}^{-1}$ Resolution Sum-Frequency Generation Vibrational Spectroscopy through Internal Heterodyne PhaseResolved Measurement. J. Phys. Chem. B 2016, 120, 1579-1589.

(16) Yamaguchi, S. Development of Single-Channel HeterodyneDetected Sum Frequency Generation Spectroscopy and Its Application to the Water/Vapor Interface. J. Chem. Phys. 2015, 143, No. 034202.

(17) Wang, J.; Bisson, P. J.; Marmolejos, J. M.; Shultz, M. J. Measuring Complex Sum Frequency Spectra with a Nonlinear Interferometer. J. Phys. Chem. Lett. 2016, 7, 1945-1949.

(18) Xu, B.; Wu, Y.; Sun, D.; Dai, H.-L.; Rao, Y. Stabilized Phase Detection of Heterodyne Sum Frequency Generation for Interfacial Studies. Opt. Lett. 2015, 40, 4472-4475.

(19) Thamer, M.; Campen, R. K.; Wolf, M. Detecting Weak Signals from Interfaces by High Accuracy Phase-Resolved Sfg Spectroscopy. Phys. Chem. Chem. Phys. 2018, 20, 25875-25882.

(20) Vanselous, H.; Petersen, P. B. Extending the Capabilities of Heterodyne-Detected Sum-Frequency Generation Spectroscopy: Probing Any Interface in Any Polarization Combination. J. Phys. Chem. C 2016, 120, 8175-8184.
(21) Wang, H.; Gao, T.; Xiong, W. Self-Phase-Stabilized Heterodyne Vibrational Sum Frequency Generation Microscopy. ACS Photonics 2017, 4, 1839-1845.

(22) Covert, P. A.; FitzGerald, W. R.; Hore, D. K. Simultaneous Measurement of Magnitude and Phase in Interferometric SumFrequency Vibrational Spectroscopy. J. Chem. Phys. 2012, 137, No. 014201.

(23) Inoue, K.-i.; Nihonyanagi, S.; Singh, P. C.; Yamaguchi, S.; Tahara, T. 2d Heterodyne-Detected Sum Frequency Generation Study on the Ultrafast Vibrational Dynamics of $\mathrm{H} 2 \mathrm{o}$ and Hod Water at Charged Interfaces. J. Chem. Phys. 2015, 142, 212431.

(24) Nihonyanagi, S.; Kusaka, R.; Inoue, K.-i.; Adhikari, A.; Yamaguchi, S.; Tahara, T. Accurate Determination of Complex X (2) Spectrum of the Air/Water Interface. J. Chem. Phys. 2015, 143, 124707.

(25) Sun, S.; Liang, R.; Xu, X.; Zhu, H.; Shen, Y. R.; Tian, C. Phase Reference in Phase-Sensitive Sum-Frequency Vibrational Spectroscopy. J. Chem. Phys. 2016, 144, 244711.

(26) Smit, W. J.; Tang, F.; Nagata, Y.; Sánchez, M. A.; Hasegawa, T.; Backus, E. H.; Bonn, M.; Bakker, H. J. Observation and Identification of a New Oh Stretch Vibrational Band at the Surface of Ice. J. Phys. Chem. Lett. 2017, 8, 3656-3660.

(27) Nojima, Y.; Suzuki, Y.; Takahashi, M.; Yamaguchi, S. Proton Order toward the Surface of Ice Ih Revealed by Heterodyne-Detected Sum Frequency Generation Spectroscopy. J. Phys. Chem. Lett. 2017, 8, 5031-5034.

(28) Wang, J.; Bisson, P. J.; Marmolejos, J. M.; Shultz, M. J. Nonlinear Interferometer: Design, Implementation, and PhaseSensitive Sum Frequency Measurement. J. Chem. Phys. 2017, 147, No. 064201.

(29) Mondal, J. A.; Nihonyanagi, S.; Yamaguchi, S.; Tahara, T. Structure and Orientation of Water at Charged Lipid Monolayer/ Water Interfaces Probed by Heterodyne-Detected Vibrational Sum Frequency Generation Spectroscopy. J. Am. Chem. Soc. 2010, 132, 10656-10657.

(30) Bain, C. D.; Barry, T. E.; Tao, Y.-T.; Evall, J.; Whitesides, G. M.; Nuzzo, R. G. Formation of Monolayer Films by the Spontaneous Assembly of Organic Thiols from Solution onto Gold. J. Am. Chem. Soc. 1989, 111, 321-335.

(31) Covert, P. A.; Hore, D. K. Assessing the Gold Standard: The Complex Vibrational Nonlinear Susceptibility of Metals. J. Phys. Chem. C 2015, 119, 271-276.

(32) Buck, M.; Eisert, F.; Grunze, M.; Trager, F. Second-Order Nonlinear Susceptibilities of Surfaces. Appl. Phys. A: Mater. Sci. Process. 1995, 60, 1-12.

(33) Dreesen, L.; Humbert, C.; Celebi, M.; Lemaire, J. J.; Mani, A. A.; Thiry, P. A.; Peremans, A. Influence of the Metal Electronic Properties on the Sum-Frequency Generation Spectra of Dodecanethiol Self-Assembled Monolayers on Pt (111), Ag (111) and $\mathrm{Au}$ (111) Single Crystals. Appl. Phys. B: Lasers Opt. 2002, 74, 621-625.

(34) Dalstein, L.; Revel, A.; Humbert, C.; Busson, B. Nonlinear Optical Response of a Gold Surface in the Visible Range: A Study by Two-Color Sum-Frequency Generation Spectroscopy. I. Experimental Determination. J. Chem. Phys. 2018, 148, 134701.

(35) Yang, W.-C.; Hore, D. K. Determining the Orientation of Chemical Functional Groups on Metal Surfaces by a Combination of Homodyne and Heterodyne Nonlinear Vibrational Spectroscopy. J. Phys. Chem. C 2017, 121, 28043-28050. 